\title{
The Efficiency of UVC Radiation in the Inactivation of Listeria monocytogenes on Beef-Agar Food Models
}

\author{
Amir M. Hamidi-Oskouei ${ }^{*}$, Christian James ${ }^{2}$ and Stephen James ${ }^{2}$ \\ ${ }^{1}$ Department of Food Manufacturing and Automation, National Centre for Food Manufacturing, \\ Holbeach Campus, University of Lincoln, Holbeach, PE12 7PT, United Kingdom \\ ${ }^{2}$ Food Refrigeration and Process Engineering Research Centre (FRPERC), Grimsby Institute (GIFHE), \\ Nuns Corner, Grimsby, North East Lincolnshire, DN34 5BQ, United Kingdom \\ Received: October 30, 2014 \\ Accepted: January 27, 2015
}

\begin{abstract}
Summary
The aim of this study is to evaluate the effect of meat content and surface smoothness on the deactivation of Listeria monocytogenes in beef-agar food models achieved by shortwave ultraviolet (UVC) light. Food models with various meat contents were made using chopped beef slices and agar solution. Prepared models together with a Listeria selective agar (LSA) plate and a slice of cooked beef were inoculated with L. monocytogenes and then exposed to UVC light. Population of Listeria reduced to below the level of detection on the LSA plates. As the content of beef in the beef-agar models increased, more L. monocytogenes cells survived. Survival was greatest on the treated cooked slice of beef. To better understand the effect of surface irregularities, a white light interferometer was used to analyse the surface smoothness of beef-agar media and LSA plates. No correlation was observed between the surface roughness of seven out of nine types of produced beef-agar media and the degree of inactivation resulting from UVC radiation at the given dose, whereas, less bacterial cells were killed as beef content of the food models increased. The findings of the current study show that the chemical composition of the treated sample also plays an important role in pathogen resistance and survival, meaning that two samples with similar surface irregularities but different chemical composition might produce very different inactivation results when exposed to UVC light.
\end{abstract}

Key words: Listeria monocytogenes, shortwave ultraviolet light (UVC), food model, white light interferometer, microbial deactivation, ready-to-eat beef, surface analysis, emerging technology, nonthermal decontamination

\section{Introduction}

Listeria monocytogenes has been identified as a significant public health risk in ready-to-eat (RTE) sliced meat products (1). This is due to the long shelf life of RTE meat products and the ability of L. monocytogenes to grow under refrigerated conditions. Contamination of RTE sliced meat products with Listeria usually occurs as a result of handling and slicing of the products following cooking and during packaging. Technologies that can eliminate L. monocytogenes from the surface of sliced meat before packaging are therefore required. One of the potential methods is ultraviolet light. Shortwave ultraviolet (UVC) radiation is a non-thermal microbial deactivation technique that does not leave any residues on the surface of treated products or produce any waste. It is an economical treatment and it can easily be installed on established production lines.

The deactivation of foodborne pathogens to below the detection limit using UVC radiation has been achieved on agar plates (2-4). Meat products including ham slices 
(5), beef slice (6), raw salmon (7), and chicken breast fillets (8) have also been treated by shortwave UV light. However, the results have not been as effective. One reason is believed to be that microorganisms are shielded by the surface irregularities on products such as beef, chicken or ham slices $(2,5,9-11)$. An alternative explanation is that the degree of survival is affected by the chemical composition of the food being treated. Bernbom et al. (12), in a study of the effect of UVC on L. monocytogenes in spent brine, used by the fish industry, concluded that the survival of the L. monocytogenes was enhanced by the presence of sodium chloride and organic food material. It was not clear whether this enhancement of survival was due to a physical protection of the cells in the food matrix or because of physiological changes in the attached bacteria, or a combination of both. However, data on the ability of UVC light to deactivate microorganism(s) on foods with different chemical or physical properties is lacking.

This study was carried out to determine whether the effectiveness of UVC against $L$. monocytogenes on meat surfaces is influenced by: (i) the chemical composition of the sample, (ii) surface irregularities of the sample being treated, or (iii) both. To achieve this goal, model products (beef-agar media) were prepared with a range of meat contents from 4.8 to $58.3 \%$ (by mass per volume). Controls were produced of Listeria selective agar (LSA) and sliced cooked beef. The surface morphology of the beef-agar media and LSA plates was examined utilising a white light interferometer (WLI), which has not been used for food surface analysis purposes extensively, and the results were correlated with the UVC-deactivation of L. monocytogenes on these media. Unfortunately, the WLI could not measure the morphology of the sliced cooked beef sample surface.

\section{Materials and Methods}

\section{Medium preparation}

Sliced roast beef was purchased from a local supermarket and chopped into small pieces (approx. 1-2 mm in all dimensions) using a hand held food blender (model 4169; Braun, Zamudio, Spain). An agar base solution was made by the addition of $1.5 \mathrm{~g}$ of agar powder (Scientific Laboratory Supplies Ltd, Manchester, UK) to $98.5 \mathrm{~mL}$ of distilled water. Nine bottles each containing the agar base solution were prepared. Differing amounts of chopped beef were added to the nine bottles to produce suspensions with different beef contents (Table 1). A magnetic stirrer was added to each bottle. The contents of each bottle were mixed on a hot plate stirrer (model 375, VWR; Radnor, PA, USA) for $30 \mathrm{~min}$ followed by sterilisation at $121{ }^{\circ} \mathrm{C}$ for $15 \mathrm{~min}$ in an autoclave (Prestige Medical, Classic bench-top, Blackburn, UK). Stirring was continued for a further $30 \mathrm{~min}$ after sterilisation and before pouring the sterile beef-agar mix into sterile Petri dishes. Approximately $20 \mathrm{~g}$ of the hot suspension (approx. $70^{\circ} \mathrm{C}$ ) was poured into each plate. Four replica plates of each meat mass per volume ratio and Listeria selective agar (LSA, CM0856; Oxoid, Hampshire, UK) plates with $0 \%$ (by mass per volume) meat were produced. Care was taken during the pouring process to ensure a consistent volume in each plate with a homogenous distribution of particles and consistent content of the beef in all plates.
Table 1. Content of beef in different beef-agar medium samples

\begin{tabular}{cc}
\hline Sample & $(m($ beef $) / V($ agar $)) / \%$ \\
\hline A5 & 4.8 \\
A10 & 9.1 \\
A20 & 16.7 \\
A40 & 28.6 \\
A60 & 37.5 \\
A80 & 44.4 \\
A100 & 50.0 \\
A120 & 54.6 \\
A140 & 58.3 \\
\hline
\end{tabular}

The poured plates were left in a refrigerator, operating at approx. $6^{\circ} \mathrm{C}$, overnight to cool and set. After setting the beef-agar plates were topped up to $(40 \pm 0.1) \mathrm{g}$ with sterile agar base solution and then returned to the refrigerator for another $3 \mathrm{~h}$. LSA plates containing $(40 \pm 0.1) \mathrm{g}$ of agar were also poured. During the production of the plates the chopped beef particles settled to the bottom of the plates. The set media were therefore inverted and aseptically inserted into sterile Petri dishes prior to UVC treatment.

\section{Inoculation of beef-agar and LSA plates}

An overnight inoculum of L. monocytogenes strain ATCC 19111 (purchased from National Centre for Type Cultures, Salisbury, UK) was prepared by adding few colonies from stock culture into $10 \mathrm{~mL}$ of tryptone soya broth (casein soya bean digest medium, Oxoid) bottles and incubating at $35{ }^{\circ} \mathrm{C}$ for $(24 \pm 2) \mathrm{h}$. Beef-agar media and LSA plates were dried at $35^{\circ} \mathrm{C}$ for $30 \mathrm{~min}$ before inoculation to remove any condensation from the surface. A volume of $0.1 \mathrm{~mL}$ of the overnight L. monocytogenes inoculum was deposited on top of each plate and spread using a sterile spreader. Four plates of each meat mass per volume ratio (40 plates in total) were inoculated, three of which were exposed to UVC radiation in the next stage and one was used as control sample. The plates were left (lid-on) at an ambient temperature of approx. $22{ }^{\circ} \mathrm{C}$ for $60 \mathrm{~min}$ to dry and for L. monocytogenes to adhere.

\section{Inoculation of beef slices}

Using a Petri dish $(r=5 \mathrm{~cm})$ as a mould, beef slices were cut to have the same exposed surface area $\left(78.5 \mathrm{~cm}^{2}\right)$ as the LSA and beef-agar medium plates. The same $L$. monocytogenes culture and the same inoculation method used for inoculating beef-agar medium and LSA plates were also used to inoculate four beef slices (three for treatment and one as control sample). Before UVC treatment, the inoculated slices were left at ambient temperature of $22{ }^{\circ} \mathrm{C}$ for $1 \mathrm{~h}$ to air-dry and for L. monocytogenes cells to adhere to the surface.

\section{UVC treatment chamber}

The UVC treatment chamber (Fig. 1) consisted of a stainless steel box $(75 \mathrm{~cm} \times 93 \mathrm{~cm} \times 104 \mathrm{~cm})$ with eight germicidal UVC bulbs (TUV PL-L 95 W; Philips, Pabianice, 


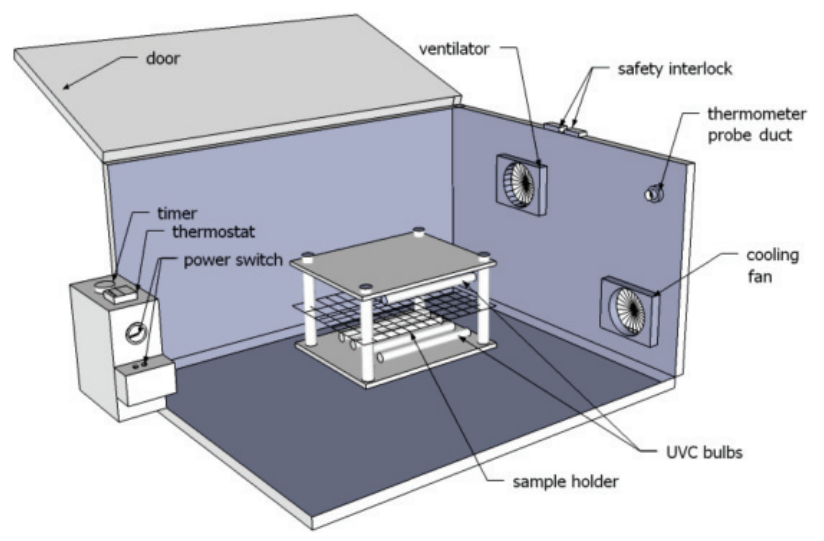

Fig. 1. Schematic illustration of UVC treatment chamber

Poland). Four of the bulbs were placed in the top and other four at the base of the box. A welded wire-mesh sample holder with $4 \mathrm{~cm}^{2}$ grid squares was installed between the emitters. The distance of the sample from the bulbs could be adjusted to achieve different field intensities. The UVC chamber was also equipped with two fans, operated via a thermostat, that removed the excess heat produced by UVC emitters, as well as an analogue timer (H3CR; Omron, Milton Keynes, UK), to control treatment time.

\section{UVC treatment}

Before each trial, the UVC emitter bulbs were preheated for $10 \mathrm{~min}$ to stabilise the UVC emission. The sample support shelf was adjusted to be $5 \mathrm{~cm}$ below the upper UVC tubes. UVC peak intensity and dosage (flux) were measured using a digital UV metre (UVICURE ${ }^{\circledR}$ Plus II, Eit, Sterling, VA, USA). Preliminary tests showed no significant difference in the measured UVC radiation at different points over the surface of the sample holder tray.

Three inoculated LSA plates were exposed to $(195 \pm 11)$ $\mathrm{mJ} / \mathrm{cm}^{2}$ of UVC radiation in the treatment chamber. This experiment was repeated for beef slice and beef-agar media with different beef contents (in \%, by mass per volume), i.e. three samples from each type at a time. Preliminary tests with higher doses reduced the population of L. monocytogenes to below the detection limit on most of the samples.

\section{Recovery of Listeria monocytogenes from control and treated samples}

After treatment each sample was removed from the Petri dish using a sterile scalpel and transferred aseptically to a filter stomacher bag (Stomacher Bags, $3500 \mathrm{~mL}$; Seward Ltd, Worthing, West Sussex, UK). To each stomacher bag $40 \mathrm{~mL}$ of buffered peptone water (BPW, CM0509; Oxoid) were added followed by stomaching (stomacher 400 circulator; Seward Ltd) for $60 \mathrm{~s}$ at $300 \mathrm{rpm}$. Diluting the samples was continued by conducting decimal dilutions in BPW followed by plating $0.1-\mathrm{mL}$ aliquots on LSA plates. The plates were then incubated for $48 \mathrm{~h}$ at $35^{\circ} \mathrm{C}$, followed by enumeration.

\section{Surface topography of the beef-agar media and LSA plates}

To measure the surface roughness of the beef-agar media and LSA plates, a white light interferometer (New
View 500; WLI; Zygo Ltd, Middlefield, CT, USA) and MetroPro software (Zygo Ltd) were utilised. A WLI is a machine which provides optical measurement for the depth of the irregularities on the sample surface. The WLI was set up using a $\times 2.5$ Mirau lens with a zoom of $\times 1.00$ and working distance of $10.3 \mathrm{~mm}$. Sa and Ra roughness parameters for tested media were determined using the MetroPro software. Ra is the arithmetic average of the absolute values along a single specified direction, and Sa is the arithmetic average of the absolute values over the whole measured area (13).

The surface roughness of each medium was measured in triplicates and $10 \mathrm{Ra}$ values were obtained for the surface of each replicate (30 readings for each sample). Initially, it was not possible to get any reading for the surface of LSA plate using the WLI, as the medium was too transparent. To overcome this, $5 \mathrm{~mL}$ of ready-mixed white paint (300 mL; Wilko Colour Time, China) were added to $500 \mathrm{~mL}$ of the LSA solution, to make the mix opaque. The solution was then poured into Petri dishes after being mixed properly. The plates were left to set before being studied by WLI. Unfortunately, the WLI instrument was unable to provide any data regarding the surface topography of beef slices possibly due to the inability of the beef slice to reflect light.

Colony forming units (CFU) of L. monocytogenes were calculated and then log transformed in Excel (Microsoft Excel, Microsoft Corporation, Redmond, WA, USA). To determine the error bars on the graphs a $95 \%$ confidence level was used. Further statistical analysis of the data to determine any probable significant difference between calculated values was carried out in SPSS (IBM SPSS Statistics v. 19; IBM Company, Armonk, NY, USA) using one-way analysis of variance (ANOVA) followed by Scheffe's post-hoc test.

\section{Results}

\section{UVC-deactivation of Listeria monocytogenes on LSA, beef-agar and beef slices}

Initial population of the L. monocytogenes on LSA plates, beef-agar medium plates and beef slices was 5.54, 5.89, and $6.19 \log \mathrm{CFU} / \mathrm{cm}^{2}$, respectively. The detection limit was $1.01 \log \mathrm{CFU} / \mathrm{cm}^{2}$. A UVC radiation dose of $(195 \pm 11) \mathrm{mJ} / \mathrm{cm}^{2}$ reduced the number of $L$. monocytogenes cells to below the detection limit in all replicates of both LSA and beef-agar media plates with 4.8, 9.1, and $16.7 \%$ (by mass per volume) beef content (Fig. 2). At beef contents above $16.7 \%$ (by mass per volume), the numbers of surviving $L$. monocytogenes tended to increase as the beef content increased (Fig. 2). Over $3 \mathrm{log} \mathrm{CFU} / \mathrm{cm}^{2}$ of L. monocytogenes cells survived on the beef-agar media with beef contents above $54.6 \%$ (by mass per volume).

The application of a UVC radiation dose of $(195 \pm 11)$ $\mathrm{mJ} / \mathrm{cm}^{2}$ produced reductions ranging from 2.95 to over $5.53 \log \mathrm{CFU} / \mathrm{cm}^{2}$ on beef-agar media with various meat contents. However, the same dosage on the beef slices only reduced the L. monocytogenes numbers from 6.19 to $5.32 \log \mathrm{CFU} / \mathrm{cm}^{2}$, i.e. a $0.87 \log$ reduction. 


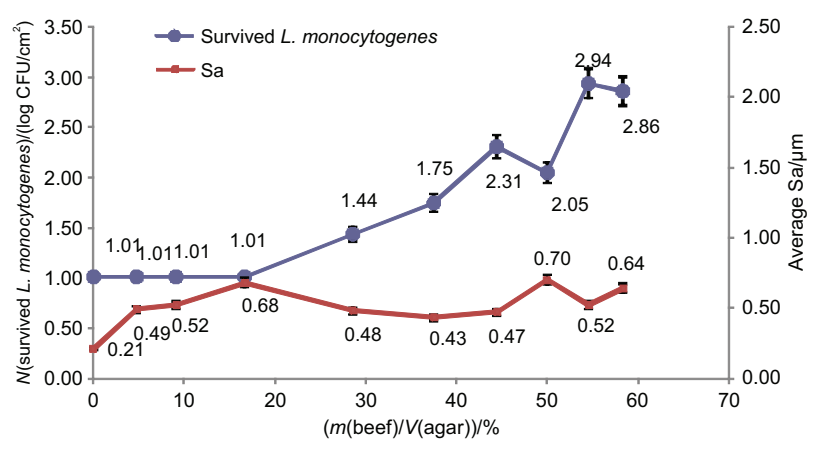

Fig. 2. Relationship between beef content in beef-agar media and levels of L. monocytogenes after UVC light treatment, and surface roughness (Sa). Beef content of zero represents Listeria selective agar (LSA) plates. Error bars indicate $95 \%$ confidence interval. Detection limit was $1 \log \mathrm{CFU} / \mathrm{cm}^{2}$

\section{Surface topography of LSA and beef-agar plates}

An example of the pictorial output of the WLI system is shown in Fig. 3. This picture shows the results of surface analysis of beef-agar with $55 \%$ (by mass per volume) beef, provided by the MetroPro software. The average of the Sa $(\mu \mathrm{m})$ values with $95 \%$ confidence intervals are shown in Fig. 2. The lowest Sa $(0.21 \mu \mathrm{m})$ value was obtained from the LSA plates, which confirms that, as expected, this sample had the smoothest surface.

An analysis of variance (ANOVA) using IBM SPSS software v. 19 showed that the LSA plates' surfaces were significantly $(p<0.05)$ smoother than the beef-agar media plates. The Sa values of the beef-agar media plates fluctuated between 0.43 and $0.70 \mu \mathrm{m}$. Post-hoc test revealed an appreciable difference $(\mathrm{p}<0.05)$ between the surface irregularities of medium containing $50 \%$ (by mass per volume) beef and the media with 4.8, 28.6, 37.5 and $44.4 \%$ (by mass per volume) beef content. This increase in the roughness of medium with 50 \% (by mass per volume) beef correlates with a decrease in the survival rate of $L$. monocytogenes cells shown in Fig. 2.

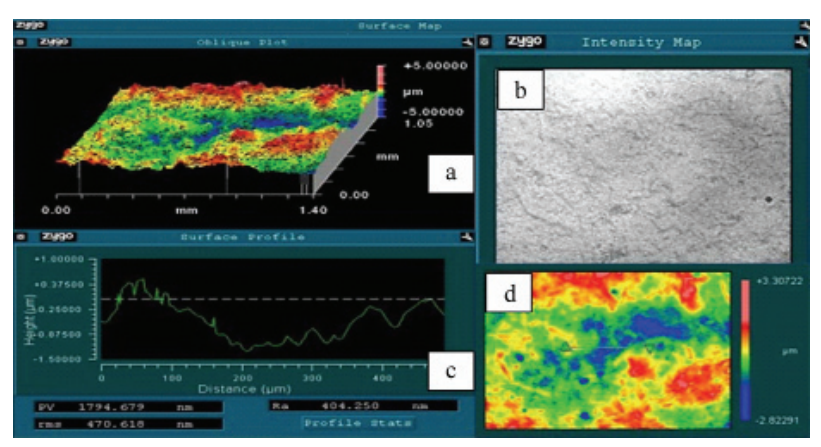

Fig. 3. Surface morphology of A120 beef-agar medium. Pictures $\mathrm{a}$ and $\mathrm{b}$ provide 3-dimensional and 2-dimensional drawing of the surface of A120, respectively. Picture c shows a graph which represents information regarding the depth of the surface irregularities over a certain distance (height $\times$ distance). Picture c also provides surface roughness ( $\mathrm{Ra}$, in $\mu \mathrm{m})$ value of the surface of the sample along the line drawn in the middle of picture d. Surface roughness over whole measured area (Sa, in $\mu \mathrm{m})$ values presented in this paper were calculated by averaging $10 \mathrm{Ra}$ readings obtained from drawing 10 random lines over picture $d$
The survival rate of L. monocytogenes improved as the beef content in the media increased. However, although a significant difference was seen between surface roughness of some of the beef-agar media at different beef mass per volume ratios, the WLI analysis did not show a steady enhancement in the general trend of the surface roughness as the survival rate of L. monocytogenes increased $\left(R^{2}=0.24\right)$. The relationship between the survival of L. monocytogenes after treatment and the Sa value is shown in Fig. 4. No obvious trend was revealed and $R^{2}(0.09)$ and root mean square error (RMSE) (2.10) values calculated using Microsoft Excel of fit of the trendline on the data show a very poor relationship.

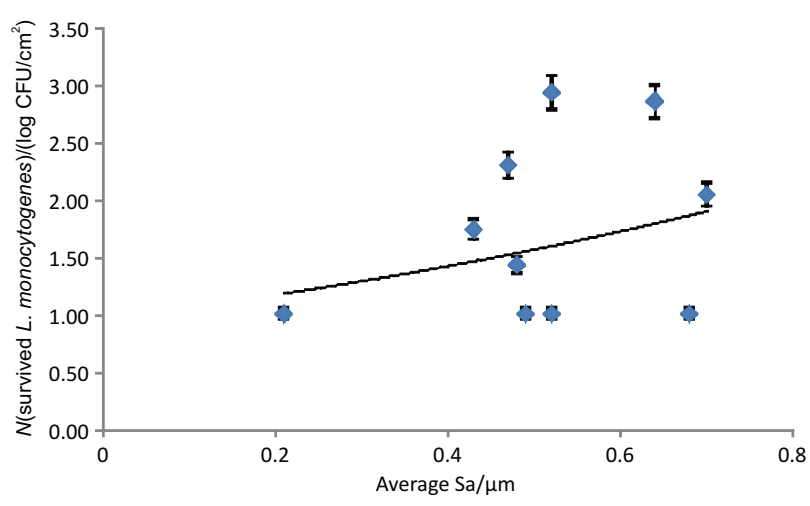

Fig. 4. Relationship between the surface roughness (Sa) of beef-agar media plates and survival rate of L. monocytogenes. Root Mean Square Error (RMSE) and $\mathrm{R}^{2}$ values are 2.1 and 0.09 , respectively. Error bars indicate $95 \%$ confidence interval

\section{Discussion}

The results obtained in the current study have shown that the efficiency of UVC in deactivating L. monocytogenes cells on the surface of agar plates decreases as the content of meat in the plates is increased.

Due to the impenetrability of UVC light, surface roughness has been suggested to be responsible for shielding microorganisms and hindering their deactivation $(2,5,11,14-16)$. It is known that the physical state of the food plays an important role in other decontamination techniques such as those based on thermal treatments (17). Studies using lux gene technologies showed substantial differences in the recovery of Salmonella typhimurium DT104 on the chicken surfaces with and without skin after the same heat treatment $(18,19)$. However, no research has previously been conducted on the effect of a measure of surface topography on microbial inactivation by UVC radiation. In this trial beef agar medium plates with various meat contents but similar surface properties were prepared to challenge the impact of 'surface irregularity' factor on UVC treatment process. No correlation was found between the degree of deactivation and the surface roughness of the plates containing 4.8, 9.1, 16.7, 28.6, 37.5, 44.4 and $54.6 \%$ (by mass per volume) beef, as measured by a WLI, thus indicating that, in this case, the degree of effectiveness was more related to the chemical composition. 
Product composition, solid content, colour, starch, and the overall chemistry of the food have been reported to have a key effect on the efficacy of UVC treatments but the effects of these individual factors are not known (20). Organic materials have been known to absorb UVC radiation (21). It was speculated by Bernbom et al. (12) that the presence of sodium chloride and organic materials impacts the efficiency of UVC radiation by absorption of the UVC rays, physical protection of the microorganism, and/ or possible induction of physiological changes in the microorganism, resulting in enhanced survivability.

The presence of organic materials has been found to reduce the efficiency of other microbial decontamination techniques. Oteiza et al. (22) studied the heat resistance of two strains of Escherichia coli following addition of fat and starch to heating menstruum prepared from morcilla (a blood sausage). The authors concluded that when fat or starch contents are increased to obtain better yields, the risk to consumers increases since both components have a protective effect on the thermal inactivation of the microorganisms. In Bermúdez-Aguirre and Barbosa-Cánovas (23) study on the inactivation of L. innocua by thermo-sonication it was reported that inactivation of Listeria cells occurs first in fat-free milk, and that the rate of inactivation decreases with increasing fat content.

WLI is a powerful tool for optically measuring the depth of roughness on the sample surface. This device was, however, unable to analyse the surface topography of beef slice and un-manipulated LSA plates possibly owing to the incapability of such surfaces to reflect light. The fluctuation in the roughness measurement results implies the limitation of this machine in measuring surface imperfections of food products. In this study, no relationship was found between the surface roughness of seven out of nine types of produced beef-agar media and the degree of inactivation resulting from UVC radiation at the given dose. However, this is the first time that the authors have read about this technique being used on a food surface. Further studies need to be carried out using the system on different food surfaces that are known to be amenable to UVC decontamination technologies.

The results of this study show that the effects of the different beef components and their contents on resistance of L. monocytogenes against UVC radiation need to be further investigated. In addition, more research needs to be done on the survival characteristics of spoilage or pathogenic microorganisms when exposed to UVC radiation on surfaces with different chemical and physical characteristics.

\section{Conclusions}

Although surface irregularities of ready-to-eat meat slices have been reported to account for the limited success of UVC treatments in deactivating microorganisms on such surfaces, the findings of the current study indicate that chemical composition may play a more important role in microbial survival/resistance than surface roughness. This could be through: (i) physical protection of the microorganisms, (ii) absorption of the UVC wavelengths by the chemical compounds present, or (iii) inducing physiological changes in the microorganisms making them resistant against UVC light. Further studies are required to identify the cause for microbial survival of microorganisms treated with UV on such food surfaces and how the effectiveness of such treatments can be improved.

\section{Acknowledgement}

We would like to acknowledge UV Technology Ltd. and Air Hygiene UK (Bristol) for providing equipment needed for manufacturing UVC treatment chamber.

\section{References}

1. Food Standards Agency. Annual Report of the Chief Scientist 2012/13. London, UK: Food Standards Agency; 2013. http://www.food.gov.uk/

2. Sumner SS, Wallner-Pendleton EA, Froning GW, Stetson LVE. Inhibition of Salmonella typhimurium on agar medium and poultry skin by ultraviolet energy. J Food Prot. 1996; 59(3):319-21.

http://www.ingentaconnect.com/content/iafp/jfp

3. Wong E, Linton RH, Gerrard DE. Reduction of Escherichia coli and Salmonella senftenberg on pork skin and pork muscle using ultraviolet light. Food Microbiol. 1998;15(4):415-23. http://dx.doi.org/10.1006/fmic.1998.0185

4. Yaun BR, Sumner SS, Eifert JD, Marcy JE. Response of Salmonella and Escherichia coli O157:H7 to UV energy. J Food Prot. 2003;66(6):1071-3.

http://www.ingentaconnect.com/content/iafp/jfp

5. Chun H, Kim J, Chung K, Won M, Song KB. Inactivation kinetics of Listeria monocytogenes, Salmonella enterica serovar Typhimurium, and Campylobacter jejuni in ready-to-eat sliced ham using UV-C irradiation. Meat Sci. 2009;83:599603.

http://dx.doi.org/10.1016/j.meatsci.2009.07.007

6. Kaess G, Weidemann JF. Effects of ultraviolet irradiation on the growth of microorganisms on chilled beef slices. Int J Food Sci Technol. 1973;8:59-69. http://dx.doi.org/10.1111/j.1365-2621.1973.tb01689.x

7. Ozer NP, Demirci A. Inactivation of Escherichia coli O157:H7 and Listeria monocytogenes inoculated on raw salmon fillets by pulsed UV-light treatment. Int J Food Sci Technol. 2006; 41:354-60. http://dx.doi.org/10.1111/j.1365-2621.2005.01071.x

8. Lyon SA, Fletcher DL, Berrang ME. Germicidal ultraviolet light to lower numbers of Listeria monocytogenes on broiler breast fillets. Poult Sci. 2007;86:964-7. http://dx.doi.org/10.1093/ps/86.5.964

9. Bintsis T, Litopoulou-Tzanetaki E, Robinson RK. Existing and potential applications of ultraviolet light in the food industry - a critical review. J Sci Food Agric. 2000;80(6):637-45. http://dx.doi.org/10.1002/(sici)1097-0010(20000501)80:6<637: :aid-jsfa603>3.0.co;2-1

10. Kowalski W. Ultraviolet germicidal irradiation handbook: UVGI for air and surface disinfection. Berlin, Germany: Springer; 2009.

11. Haughton PN, Lyng J, Cronin D, Fanning S, Whyte P. Effect of crust freezing applied alone and in combination with ultraviolet light on the survival of Campylobacter on raw chicken. Food Microbiol. 2012;32(1):147-51. http://dx.doi.org/10.1016/j.fm.2012.05.004

12. Bernbom N, Vogel BF, Gram L. Listeria monocytogenes survival of UV-C radiation is enhanced by presence of sodium 
chloride, organic food material and by bacterial biofilm formation. Int J Food Microbiol. 2011;147(1):69-73.

http://dx.doi.org/10.1016/j.ijfoodmicro.2011.03.009

13. Waugh DG, Lawrence J, Brown EM. Osteoblast cell response to a $\mathrm{CO}_{2}$ laser modified polymeric material. Opt Laser Eng. 2012;50:236-47.

http://dx.doi.org/10.1016/j.optlaseng.2011.08.010

14. Yaun BR, Sumner SS, Eifert JD, Marcy JE. Inhibition of pathogens on fresh produce by ultraviolet energy. Int J Food Microbiol. 2004;90:1-8.

http://dx.doi.org/10.1016/s0168-1605(03)00158-2

15. Chun HH, Kim JY, Lee BD, Yu DJ, Song KB. Effect of UV-C irradiation on the inactivation of inoculated pathogens and quality of chicken breasts during storage. Food Control. 2010;21:276-80.

http://dx.doi.org/10.1016/j.foodcont.2009.06.006

16. Bermúdez-Aguirre D, Barbosa-Cánovas GV. Disinfection of selected vegetables under nonthermal treatments: chlorine, acid citric, ultraviolet light and ozone. Food Control. 2013; 29:82-90.

http://dx.doi.org/10.1016/j.foodcont.2012.05.073

17. Orta-Ramirez A, Marks BP, Warsow CR, Booren AM, Ryser ET. Enhanced thermal resistance of Salmonella in whole muscle compared to ground beef. J Food Sci. 2005;70(7):35962.

http://dx.doi.org/10.1111/j.1365-2621.2005.tb11480.x
18. James SJ, Evans JA. Predicting the reduction in microbes on the surface of foods during surface pasteurisation - the 'BUGDEATH' project. J Food Eng. 2006;76(1):1-6. http://dx.doi.org/10.1016/j.jfoodeng.2005.05.011

19. Lewis RJ, Robertson K, Alloush HA, Dowman T, Salisbury V. Use of bioluminescence to evaluate the effects of rapid cooling on recovery of Salmonella enterica serovar Typhimurium DT104 after heat treatment. J Food Eng. 2006;76(1):49-52. http://dx.doi.org/10.1016/j.jfoodeng.2005.05.013

20. Sastry SK, Datta AK, Worobo RW. Ultraviolet light. J Food Sci. 2000;65(12):90-2. http://dx.doi.org/10.1111/j.1750-3841.2000.tb00623.x

21. Andersen BM, Banrud H, Boe E, Bjordal O, Drangsholt F. Comparison of UVC light and chemicals for disinfection of surfaces in hospital isolation units. Infect Control Hosp Epidemiol. 2006;27:729-34. http://dx.doi.org/10.1086/503643

22. Oteiza JM, Giannuzzi L, Califano AN. Thermal inactivation of Escherichia coli O157: H7 and Escherichia coli isolated from morcilla as affected by composition of the product. Food Res Int. 2003;36(7):703-12. http://dx.doi.org/10.1016/s0963-9969(03)00050-4

23. Bermúdez-Aguirre D, Barbosa-Cánovas GV. Study of butter fat content in milk on the inactivation of Listeria innocua ATCC 51742 by thermo-sonication. Innovative Food Sci Emerging Technol. 2008;9(2):176-85. http://dx.doi.org/10.1016/j.ifset.2007.07.008 